\title{
Sampling Trajectories for Mobile Sensing
}

\author{
Jayakrishnan Unnikrishnan and Martin Vetterli \\ School of Computer and Communication Sciences \\ Ecole Polytechnique Fédérale de Lausanne (EPFL), CH-1015 Lausanne, Switzerland \\ Email: \{jay.unnikrishnan, martin.vetterli\}@epfl.ch
}

\begin{abstract}
Classical sampling theory for sampling and reconstructing bandlimited fields in $\mathbb{R}^{d}$ addresses the problem of sampling on lattice points. We consider a generalization of this problem, in which one samples the field along 1-dimensional spatial trajectories in $\mathbb{R}^{d}$ rather than at points. The process of sampling records the value of the field at all points on the sampling trajectories. Such a sampling setup is relevant in the problem of spatial sampling using mobile sensors. We study various possible designs of sampling trajectories and discuss necessary and sufficient conditions for perfect reconstruction. We introduce a density metric for trajectories which we call path density, that quantifies the total length of these trajectories per unit volume in $\mathbb{R}^{d}$. We formulate the problem of identifying optimal sampling trajectories that admit perfect reconstruction of bandlimited fields and are minimal in terms of the path density metric. We identify optimal sampling trajectories from a restricted class of trajectories.
\end{abstract}

\section{INTRODUCTION}

Consider the problem of sampling a $d$-dimensional timeinvariant spatial field $f(r)$ where $r \in \mathbb{R}^{d}$. If $f$ is bandlimited, results from classical sampling theory (see e.g., [1], [2]) provide schemes for sampling and reconstructing the field based on measurements of the field at a countable number of spatial locations situated on a lattice. These results are all motivated by the fact that one typically employs static sensors to measure the field at their locations.

The scenario is a little different in the problem of sampling a $d$-dimensional time-invariant spatial field (where $d=2$ or 3 ) using mobile sensors that move along fixed continuous paths through space and take measurements along their path. Mobile sensing has the advantage that a single sensor can be used to take measurements at several positions within an area of interest. Moreover, it is often inexpensive to sample the field along the sensors' paths at very high spatial frequencies. This has been noted to lead to a significant reduction in the amount of spatial aliasing in some applications [3]. Furthermore, as we point out in [4], a moving sensor admits filtering over space in the direction of motion of the sensor whereas no such spatial filtering is possible in the case of static sampling.

Since it is inexpensive to increase the sampling rate employed by the moving sensors it is reasonable to assume that the sensors can record the field values at all points along

This research was supported by ERC Advanced Investigators Grant: Sparse Sampling: Theory, Algorithms and Applications SPARSAM no 247006.
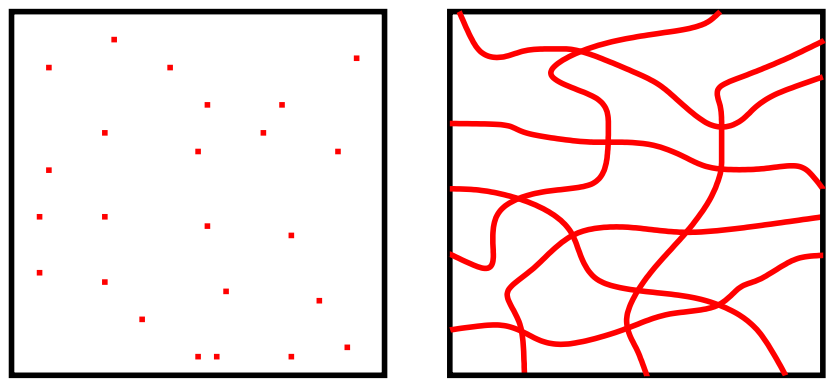

Fig. 1. Sampling a field in $\mathbb{R}^{2}$ on points and curves.

their paths. Therefore it is of interest to study schemes to reconstruct the entire $d$-dimensional field using the values of the field along the 1-dimensional curves representing the paths of the sensors through $\mathbb{R}^{d}$. Such problems call for a generalization of the classical theory of sampling of $d$ dimensional fields on countable sets of points to sampling on countable sets of 1-dimensional trajectories on $\mathbb{R}^{d}$. The two different scenarios are illustrated in Figure 1 for 2dimensional fields. The first drawing depicts the conventional sampling scheme of sampling on points while the second drawing depicts sampling on one-dimensional curves on $\mathbb{R}^{2}$.

As a generalization of classical results in sampling theory, we identify some sampling paths that admit perfect reconstruction of bandlimited fields. We introduce a metric for sampling trajectories as a natural generalization of the sampling density metric in classical sampling. The new metric which we call the path density can be interpreted as the average distance that needs to be traveled by the sensors per unit $d$-dimensional volume of the field being sampled. We formulate the problem of designing sampling trajectories that are minimal in the path density metric among all trajectories that admit perfect reconstruction of bandlimited fields. We provide a partial solution to this problem.

The literature on the design of sampling trajectories is quite limited. In the past, some schemes for reconstructing isotropic fields based on measurements taken along circular trajectories have been proposed [5], [6]. Various sampling trajectories have also been studied in the context of Magnetic Resonance Imaging (see, e.g., [7]). However to the best of our knowledge this paper is the first to explicitly address the problem of designing trajectories for general bandlimited fields and to introduce the notion of optimal trajectories for 
sampling and reconstructing bandlimited fields.

We will use the following notations for order statistics. For a function $h($.$) of a real variable we say h(a) \in \mathrm{O}(a)$ if there exists $k, A \in \mathbb{R}$ such that for all $a \geq A$, we have,

$$
|h(a)| \leq k a .
$$

Similarly, we say $h(a) \in \mathrm{o}(a)$ if for every for $\epsilon>0$ there exists $A \in \mathbb{R}$ such that and for all $a \geq A$, we have,

$$
|h(a)| \leq \epsilon a .
$$

The rest of this paper is organized as follows. In Section II we describe several possible sampling strategies and provide necessary and sufficient conditions for perfect reconstruction of bandlimited fields. We discuss the design of optimal sampling trajectories in Section III and reconstruction schemes in Section IV. We summarize our results and discuss avenues for future work in Section V. The proofs of most results are provided in the appendix. However, the proofs of the main Theorems 2.4 and 3.1 have been left out due to the lack of space and will be provided in the journal version of the paper [8].

\section{SAMPLING TRAJECTORIES}

\section{A. Preliminaries}

We will use the following terminology in this paper. A trajectory $p_{i}$ in $\mathbb{R}^{d}$ refers to a curve in $\mathbb{R}^{d}$. We represent a trajectory by a continuous function $p_{i}($.$) of a real variable$ taking values on $\mathbb{R}^{d}$ :

$$
p_{i}: \mathbb{R} \mapsto \mathbb{R}^{d} .
$$

A trajectory set $p$ is defined as a countable collection of trajectories:

$$
p:=\left\{p_{i}: i \in \mathbb{I}\right\}
$$

where $\mathbb{I} \subseteq \mathbb{Z}$ and for each $i \in \mathbb{I}, p_{i}$ is a trajectory in the trajectory set $p$. The path density $\ell(p)$ of a given trajectory set $p$ is defined as follows:

$$
\ell(p):=\limsup _{a \rightarrow \infty} \frac{\mathcal{D}^{p}(a)}{\operatorname{Vol}_{d}(a)}
$$

where $\mathcal{D}^{p}(a)$ represents the total arc-length of trajectories from $p$ located within a $d$-dimensional spherical ball of radius $a$ centered at the origin in $\mathbb{R}^{d}$, and $\operatorname{Vol}_{d}(a)$ represents the volume of the ball. Clearly, $\mathrm{Vol}_{2}(a)=\pi a^{2}$ and $\mathrm{Vol}_{3}(a)=$ $\frac{4}{3} \pi a^{3}$. For a trajectory set composed of differentiable functions $p_{i}($.$) we note that \mathcal{D}^{p}(a)$ can be explicitly defined as

$$
\mathcal{D}^{p}(a)=\sum_{i \in \mathbb{I}} \int_{t \in T_{i}(a)}\left\|\frac{d p_{i}(t)}{d t}\right\| d t
$$

where $\|x\|$ represents the Euclidean norm of $x \in \mathbb{R}^{d}$ and

$$
T_{i}(a):=\left\{t \in \mathbb{R}:\left\|p_{i}(t)\right\| \leq a\right\}
$$

represents the portion of trajectory $p_{i}($.$) that lies within the$ ball of radius $a$ centered at the origin.

A $d$-dimensional field $f$ is a real-valued function defined on $\mathbb{R}^{d}$

$$
f: \mathbb{R}^{d} \mapsto \mathbb{R} .
$$

For a square integrable $d$-dimensional field $f \in L^{2}\left(\mathbb{R}^{d}\right)$ we define its Fourier transform $F$ as

$$
F(\omega):=\int_{\mathbb{R}^{d}} f(r) \exp (-\mathrm{i}\langle\omega, r\rangle) d r, \quad \omega \in \mathbb{R}^{d}
$$

where $\mathrm{i}$ denotes the imaginary unit, and $\langle u, v\rangle$ denotes the inner product between vectors $u$ and $v$. Let $\mathcal{B}_{\mho}$ denote the collection of fields $f$ such that the Fourier transform $F$ of $f$ is supported on the interior of a set $\mho \subset \mathbb{R}^{d}$. For $f \in \mathcal{B}_{\mho}$ we say that $f$ is bandlimited to $\mho$. Let $\mathcal{C}_{\mho}$ denote the collection of all trajectory sets $p$ that satisfy the following conditions:

(C1) Any field $f \in \mathcal{B}_{\mho}$ can be reconstructed exactly from its values on the trajectories in $p$. I.e. the field $f \in \mathcal{B}_{\mho}$ is uniquely determined by the values $f\left(p_{i}(t)\right): i \in$ $\mathbb{I}, t \in \mathbb{R}$.

(C2) There is a continuous curve of length $\mathcal{D}^{p}(a)+\mathrm{o}\left(a^{d}\right)$ that contains the portion of the trajectory set $p$ that is located within a spherical ball of radius $a$ centered at the origin.

The condition (C1) ensures that the entire field can be reconstructed exactly from its values on the sampling trajectories. The condition (C2) ensures that the path density metric does indeed capture the total length that needs to be traversed by a single moving sensor using the trajectories in $p$ for sampling. This avoids degenerate static cases like the situation in which every trajectory $p_{i}$ corresponds to a single point in a classical sampling lattice for the field $f$. Such a degenerate trajectory set satisfies condition (C1) and has path density equal to zero, but any path that visits all of these points has a non-zero path density. In fact, we know from Nyquist sampling theory that if we have $\mathrm{O}\left(a^{d}\right)$ sensors available for sampling spherical regions of radius $a$, it is possible to sample bandlimited fields without any movement at all.

In a practical deployment, it is not possible to take measurements of the field at all points along a continuous path because a continuous path has an infinite number of points. However, if the sensor moving along a trajectory is exposed to a bandlimited function of time it is possible to reconstruct the entire field along its path from uniformly spaced samples. This motivates the following additional desirable condition (K1) for a trajectory set $p$ :

(K1) For any field $f \in \mathcal{B}_{\mho}$ the 1-dimensional signal $f\left(p_{i}().\right)$ is bandlimited ${ }^{1}$ for all $p_{i} \in p$.

Almost all of the trajectory sets that we study in this paper satisfy condition (K1). In the following lemma we show that all trajectory sets that satisfy this condition are made up of straight-line trajectories or points.

Lemma 2.1: Any trajectory set $p$ that satisfies condition (K1) is made up of straight line trajectories, i.e., the trajectories in $p$ can be expressed as

$$
p_{i}(t)=\left(p_{i, 1}(t), p_{i, 2}(t), \ldots, p_{i, d}(t)\right), \quad t \in \mathbb{R}, i \in \mathbb{I} \subset \mathbb{Z}
$$

\footnotetext{
${ }^{1}$ i.e. has compact support in the Fourier domain
} 


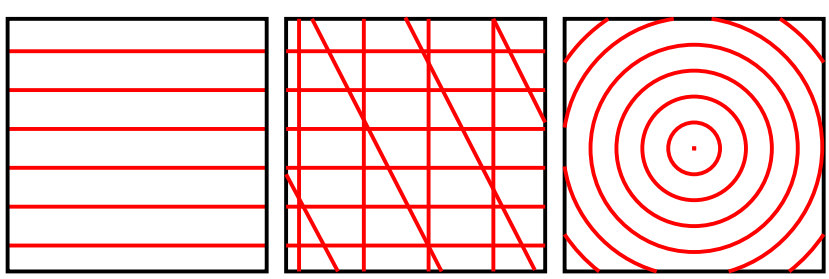

Fig. 2. Three choices of sampling trajectory sets for $\mathbb{R}^{2}$ : Set of regular parallel trajectories, union of sets of regular parallel trajectories, and concentric equispaced circular trajectories.

where $p_{i, j}(t)$ is an affine function of $t$ for each $i, j$.

We also have the following lemma which we will use later in the paper.

Lemma 2.2: Consider a field $\left\{f(r): r \in \mathbb{R}^{d}\right\}$ with Fourier transform supported on a compact set $\mho \subset \mathbb{R}^{d}$. For $r^{0}, r^{1} \in \mathbb{R}^{d}$ let $p(t):=r^{0}+r^{1} t$ denote a linear trajectory parameterized by $t$. Then the Fourier transform of the function $f(p()$.$) is bandlimited to the set [\underline{\Omega}, \bar{\Omega}]$ where

$\underline{\Omega}=\min \left\{\left\langle\omega, r^{1}\right\rangle: \omega \in \mho\right\}$ and $\bar{\Omega}=\max \left\{\left\langle\omega, r^{1}\right\rangle: \omega \in \mho\right\}$.

We provide proofs for both the above lemmas in the appendix.

\section{B. Sampling trajectories for $\mathbb{R}^{2}$}

We now present some simple examples of trajectory sets for sampling two-dimensional fields and compute their path densities. For certain types of trajectory sets we provide necessary and sufficient conditions that ensure that these trajectory sets are elements in $\mathcal{C}_{\mho}$ for specific choices of $\mho \subset \mathbb{R}^{2}$. These results can be interpreted as a generalization of known results on conditions on sampling lattices [1] and unions of sampling lattices [9] for perfect reconstruction of bandlimited fields.

1) Set of regular parallel trajectories: Consider a trajectory set $p^{\alpha}=\left\{p_{i}^{\alpha}: i \in \mathbb{Z}\right\}$ composed of equispaced lines parallel to the $\mathrm{x}$-axis given by

$$
p_{i}^{\alpha}(t)=(t, \alpha i)^{T}, i \in \mathbb{Z}, t \in \mathbb{R}
$$

where $\alpha$ denotes the separation between adjacent trajectories in this trajectory set. Such a trajectory set is the first example in Figure 2. Suppose

$$
\underline{\Omega}:=\inf _{\omega \in \mho} \omega_{y} \quad \text { and } \quad \bar{\Omega}:=\sup _{\omega \in \mho} \omega_{y} .
$$

Let $\alpha^{*}:=\frac{2 \pi}{\bar{\Omega}-\Omega}$. Then we have the following results that follow easily from classical sampling theory [1]:

$$
p^{\alpha} \in \mathcal{C}_{\mho} \text { if } \alpha<\alpha^{*}
$$

and

$$
\sup \left\{\alpha: p^{\alpha} \in \mathcal{C}_{\mho}\right\}=\alpha^{*} .
$$

Both these above results are special cases of Theorem 2.4 which we prove later in the paper. In the following lemma we characterize the path density of a regular set of parallel trajectories.

Lemma 2.3: For the trajectory set $p$ defined in (4) we have

$$
\ell(p)=\frac{1}{\alpha} .
$$

We provide a proof in the appendix.

2) Union of sets of regular parallel trajectories: For vectors $v^{0, i}, v^{1, i}, v^{2, i} \in \mathbb{R}^{2}$ such that $v^{1, i}$ and $v^{2, i}$ are noncolinear, let $p_{i}^{\alpha}$ denote the set of regular parallel trajectories defined by $p_{i}^{\alpha}=\left\{p_{i, j}^{\alpha}: j \in \mathbb{Z}\right\}$ where

$$
p_{i, j}^{\alpha}(t)=v^{0, i}+\alpha j v^{1, i}+t v^{2, i}, j \in \mathbb{Z}, t \in \mathbb{R} .
$$

We also define the vector $u^{i} \in \mathbb{R}^{2}$ as the unique vector that satisfies,

$$
\left\langle u^{i}, v^{2, i}\right\rangle=0 \text { and }\left\langle u^{i}, v^{1, i}\right\rangle=2 \pi .
$$

Suppose we have $N$ such distinct trajectory sets $\left\{p_{i}^{\alpha}: 1 \leq\right.$ $i \leq N\}$ of the form (6) such that $\left\{p_{i, j}^{\alpha}(t): j \in \mathbb{Z}, t \in \mathbb{R}\right\} \neq$ $\left\{p_{k, j}^{\alpha}(t): j \in \mathbb{Z}, t \in \mathbb{R}\right\}$ for $i \neq k$. Let $\hat{p}^{\alpha}$ denote the union of all the sets:

$$
\hat{p}^{\alpha}:=\bigcup_{i=1}^{N} p_{i}^{\alpha} .
$$

An example for such a trajectory set is the second sketch in Figure 2. It is easy to see that the separation between trajectories in the set $p_{i}^{\alpha}$ is equal to $\alpha \Delta_{i}$ where

$$
\Delta_{i}:=\left\langle v^{1, i}, \frac{u^{i}}{\left\|u^{i}\right\|}\right\rangle=\frac{2 \pi}{\left\|u^{i}\right\|} .
$$

Hence it follows from Lemma 2.3 that the path density of this trajectory set is given by

$$
\ell\left(\hat{p}^{\alpha}\right)=\sum_{i=1}^{N} \frac{1}{\alpha \Delta_{i}}=\frac{1}{2 \pi \alpha} \sum_{i=1}^{N}\left\|u^{i}\right\| .
$$

We now introduce some further notation. For $\mho \subset \mathbb{R}^{d}$ and $r \in \mathbb{R}^{d}$ we define

$$
\left.\mho(r):=\left\{x \in \mathbb{R}^{d}: x-r \in \mho\right\}\right\} .
$$

We have the following result.

Theorem 2.4: Let $\mho \subset \mathbb{R}^{2}$ be a compact convex set. Let $\hat{p}^{\alpha}$ denote the union of the sets of trajectories defined in (8). Let $\mathcal{Q}^{\alpha} \subset \mathbb{R}^{2}$ denote the set of points

$$
\mathcal{Q}^{\alpha}:=\left\{\sum_{i=1}^{N}(-1)^{n_{i}} \frac{u^{i}}{2 \alpha}: n_{i} \in\{0,1\}, 1 \leq i \leq N\right\}
$$

where $\left\{u^{i}\right\}_{1}^{N}$ are chosen to satisfy (7). Further, let

$$
\alpha^{*}:=\sup \left\{\alpha: \mathcal{Q}^{\alpha} \nsubseteq \mho(r), \text { for all } r \in \mathbb{R}^{2}\right\} .
$$

Then we have

$$
\hat{p}^{\alpha} \in \mathcal{C}_{\mho} \text { for all } \alpha \in\left(0, \alpha^{*}\right),
$$

and,

$$
\sup \left\{\alpha: \hat{p}^{\alpha} \in \mathcal{C}_{\mho}\right\}=\alpha^{*} .
$$


whence we get $\ell(c)=\frac{\Omega}{\pi}$.

Theorem 2.4 gives us necessary and sufficient conditions on unions of sets of regular parallel trajectories that admit perfect reconstruction. A proof of this result is provided in [8]. The conclusion shows that it is possible to recover the field completely using readings on multiple regular parallel trajectory sets even when the measurements on each individual regular parallel trajectory set may be aliased. Theorem 2.4 can be generalized to higher dimensions where sampling on lines is replaced with sampling on hyperplanes. Such a generalization is also considered in [8].

These results can be interpreted as a special case of sampling on a union of lattices. We know from Lemma 2.1 that for fields $f \in \mathcal{B}_{\mho}$ each of the trajectories $p_{i, j}^{\alpha}$ described in (6) the signal $f\left(p_{i, j}^{\alpha}().\right)$ is bandlimited. We also know from Lemma 2.2 that for a fixed $i$, the bandwidths of the $f\left(p_{i, j}^{\alpha}().\right)$ are identical for all $j$. Now suppose that the sensors moving along each of the trajectories in the set $p_{i}^{\alpha}$ take uniform spatial samples that are $\epsilon_{i}$ apart. From the fact that $f\left(p_{i, j}^{\alpha}().\right)$ is bandlimited, we know that, for each $i, j$, the field values $\left\{f\left(p_{i, j}^{\alpha}(t)\right): t \in \mathbb{R}\right\}$ can be recovered exactly from the samples $\left\{f\left(p_{i, j}^{\alpha}\left(m \epsilon_{i}\right)\right): m, j \in \mathbb{Z}\right\}$ provided $\epsilon_{i}$ is small enough. It is also clear that the points $\left\{p_{i, j}^{\alpha}\left(m \epsilon_{i}\right): m, j \in \mathbb{Z}\right\}$ form a lattice in $\mathbb{R}^{2}$. Thus the collection of sample locations from all the trajectory sets, i.e., the set $\left\{p_{i, j}^{\alpha}\left(m \epsilon_{i}\right): m, j \in\right.$ $\mathbb{Z}, 1 \leq i \leq N\}$ corresponds to a union of $N$ lattices. Thus the result of Theorem 2.4 can be interpreted as a result on sampling a 2-dimensional bandlimited field on a union of lattices [9] when $\epsilon_{i}$ is small enough. We note that in this example, the samples taken along each individual lattice are aliased but given all sets of samples, perfect recovery is possible.

The result of Theorem 2.4 on multiple trajectory sets is similar in spirit to Papoulis' result on generalized sampling [10]. Papoulis [10, Example 2] provides necessary and sufficient conditions for sampling on unions of shifted lattices whereas, as the arguments in the previous paragraph suggest, our results provide some conditions for sampling on unions of lattices that are not necessarily shifted versions of each other.

3) Concentric equispaced circular trajectories: Suppose $\mho$ is a disc of radius $\Omega$ centered at the origin. Let $c=\left\{c_{i}\right.$ : $i \in \mathbb{N} \cup\{0\}\}$ where $c_{i}$ denotes a circular trajectory of radius $i \frac{\pi}{\Omega}$ centered at the origin. Such a trajectory set is shown in the third sketch in Figure 2. It is known from [6] that any field bandlimited to $\mho$ is reconstructible from its values on the trajectories in set $c$. Hence condition (C1) is satisfied. It is also obvious that condition (C2) is also satisfied by this trajectory set. Thus $c \in \mathcal{C}_{\mho}$. However this trajectory set does not satisfy condition (K1) in Section II-A because the signals along the circles are not bandlimited in general. Nevertheless, it can be shown [8] that these signals have a finite essential bandwidth. We now compute the path density for this trajectory set. A disc of radius $a$ contains a total of $K \approx \frac{\Omega a}{\pi}$ concentric circles separated by radial distance of $\frac{\pi}{\Omega}$. This leads to a total trajectory length of $\frac{\pi^{2}}{\Omega} K(K+1)$

\section{Sampling trajectories for $\mathbb{R}^{d}$ where $d \geq 3$}

For $d \geq 3$, we consider only trajectory sets composed of regular parallel trajectories analogous to the set of trajectories considered in Section II-B.1 for $d=2$. Let $\left\{v^{1}, v^{2}, \ldots, v^{d}\right\}$ denote a basis for $\mathbb{R}^{d}$. Consider trajectories $p_{m}$ of the form

$$
p_{m}(t)=\sum_{i=1}^{d-1} m_{i} v^{i}+t v^{d}, \quad t \in \mathbb{R}
$$

where $m=\left(m_{1}, m_{2}, \ldots, m_{d-1}\right)^{T} \in \mathbb{Z}^{d-1}$. Let $p$ denote the trajectory set

$$
p=\left\{p_{m}: m \in \mathbb{Z}^{d-1}\right\}
$$

For $\mho \subset \mathbb{R}^{d}$, we use $\mathcal{E}_{\mho} \subset \mathcal{C}_{\mho}$ to denote the collection of all trajectory sets in $\mathcal{C}_{\mho}$ that can be expressed in the form (14). The following theorem provides sufficient conditions on the vectors $\left\{v^{1}, v^{2}, \ldots, v^{d}\right\}$ and the set $\mho$ so that $p \in \mathcal{C}_{\mho}$.

Theorem 2.5: Let $p$ denote the trajectory set defined in (14). Let $\left\{u^{1}, \ldots, u^{d-1}\right\}$ denote vectors in $\mathbb{R}^{d}$ satisfying $\left\langle u^{i}, v^{j}\right\rangle=2 \pi \delta_{i j}$ for $1 \leq i \leq d-1$ and $1 \leq j \leq d$ and let $\mho \subset \mathbb{R}^{d}$ denotes a compact convex set with a point of symmetry at the origin. Then we have $p \in \mathcal{C}_{\mho}$ if

$$
\frac{1}{2} \sum_{i=1}^{d-1} m_{i} u^{i} \notin \mho, \text { for all } m \in\{-1,0,1\}^{d-1} \backslash\{0\}^{d-1} .
$$

The condition (15) is also necessary in the sense that if there exists $m \in\{-1,0,1\}^{d-1} \backslash\{0\}^{d-1}$ such that $\frac{1}{2} \sum_{i=1}^{d-1} m_{i} u^{i} \in$ $\stackrel{\circ}{\mho}$, where $\stackrel{\circ}{\mho}$ denotes the interior of $\mho$ in $\mathbb{R}^{d}$, then $p \notin \mathcal{C}_{\mho}$.

A proof of this result is provided in [8]. The path density of the trajectory set $p$ is given in the following lemma.

Lemma 2.6: The path density of the trajectory set $p$ defined in (14) is given by

$$
\ell(p)=\frac{\left\|v^{d}\right\|}{|\operatorname{det}(G)|}
$$

where $\operatorname{det}(G)$ denotes the determinant of the Gram matrix defined by $G_{i j}=\left\langle v^{i}, v^{j}\right\rangle, 1 \leq i, j \leq d$.

We provide a proof outline in the appendix.

\section{OPTIMAL SAMPLING TRAJECTORIES}

As we argued earlier, the path density of a trajectory set captures the total distance required to be traveled per unit area for sampling spatial fields using the trajectory set. Hence it is of interest to characterize the optimal trajectory set for sampling fields that are bandlimited to a given set $\mho \subset \mathbb{R}^{d}$. We seek a solution to the following problem:

$$
\min _{p \in \mathcal{C}_{\mho}} \ell(p) \text {. }
$$

In this section we identify partial solutions to the problem, solving it exactly for trajectory sets restricted to some subsets of $\mathcal{C}_{\mho}$. 


\section{A. Optimality for $\mathbb{R}^{2}$}

It is difficult ${ }^{2}$ to characterize all the trajectory sets that satisfy conditions (C1) and (C2). However, as we show below, it is possible to identify the optimal trajectory set among those that can be written as a finite union of regular sets of parallel trajectories like in (8). Such trajectory sets have the added advantage that they satisfy the desirable property of (K1), as proved in Lemma 2.1.

Let $\mathcal{D}_{\mho} \subset \mathcal{C}_{\mho}$ denote the collection of trajectory sets $q \in$ $\mathcal{C}_{\mho}$ such that $q$ can be expressed as a finite union of the form

$$
q=\bigcup_{i=1}^{n} q^{i}
$$

where for each $i, q^{i}$ is a trajectory set of regular parallel trajectories. We need the following definitions. For a nonempty compact convex set $\mho \subset \mathbb{R}^{d}$ and any $u \in \mathbb{R}^{d}$ let $B^{u}(\mho)$ denote the distance between the two parallel supporting hyperplanes of $\mho$ that are perpendicular to the vector $u$. We refer to $B^{u}(\mho)$ as the breadth of $\mho$ in the direction $u$. The width of $\mho$ is defined by the relation

$$
\mathcal{W}(\mho):=\min _{u \in \mathbb{R}^{2}} B^{u}(\mho) .
$$

A chord of $\mho$ is defined as the nonempty intersection of $\mho$ with a line in $\mathbb{R}^{d}$. For $u \in \mathbb{R}^{d}, \mathcal{W}^{u}(\mho)$ is defined as the maximum length of a chord of $\mho$ parallel to $u$. The width $\mathcal{W}(\mho)$ can alternately also be interpreted as (see, e.g., [12])

$$
\mathcal{W}(\mho)=\min _{u \in \mathbb{R}^{2}} \mathcal{W}^{u}(\mho)
$$

We have the following result which follows easily from Theorem 2.4. We provide a proof in the appendix.

Theorem 3.1: For any nonempty compact convex set $\mho \subset$ $\mathbb{R}^{2}$, let $\hat{u}$ be the minimizer in (17), and for $\epsilon>0$ let $p^{\epsilon}$ denote a trajectory set of regular parallel trajectories given by $p^{\epsilon}=\left\{p_{j}^{\epsilon}: j \in \mathbb{Z}\right\}$ where

$$
p_{j}^{\epsilon}(t)=j\left[\left(\frac{2 \pi}{\mathcal{W}(\mho)}-\epsilon\right) \frac{\hat{u}}{\|\hat{u}\|}\right]+\hat{u}^{\perp} t, t \in \mathbb{R}, j \in \mathbb{Z}
$$

where $\hat{u}^{\perp} \in \mathbb{R}^{2}$ is orthogonal to $\hat{u}$. Then $p^{\epsilon} \in \mathcal{D}_{\mho}$ for all $\epsilon>0$ and is optimal in path density as $\epsilon \rightarrow 0$. I.e.,

$$
\lim _{\epsilon \rightarrow 0} \ell\left(p^{\epsilon}\right)=\inf _{q \in \mathcal{D}_{\mho}} \ell(q)=\frac{2 \pi}{\mathcal{W}(\mho)} .
$$

Theorem 3.1 thus establishes the optimality of a regular set of parallel trajectories $p$ from all trajectory sets in $\mathcal{D}_{\mho}$. It may be possible to demonstrate that such a trajectory set is optimal in terms of path density among a more general class of trajectory sets. A starting point for rigorously establishing such a result would be Landau's necessary conditions for sampling bandlimited functions [13] (see also [11, Corollary

\footnotetext{
${ }^{2}$ Note that even in the case of non-uniform sampling of a bandlimited function in 1-dimension, there is no known result that characterizes all collections of sampling points that admit perfect reconstruction. This is in spite of the fact that some necessary [11] conditions and sufficient [2] conditions for perfect reconstruction are known - e.g., inter-sample spacing less than Nyquist interval [2].
}

1]). However such a result is beyond the scope of this paper. Some inconclusive evidence for this conjecture is provided by our observation in [8] that there is a set of regular parallel trajectories with path density arbitrarily close to the path density of any trajectory set that visits all points in a sampling lattice for $\mho$.

We can also make a stronger conjecture related to this result. Suppose we relax condition $(\mathbf{C 2})$ so that we now allow the use of up to $N(a)$ number of mobile sensors for covering the portions of the trajectories within circular fields of radius $a$. It may be possible to show that even with this increased number of sensors, the optimality of the single set of parallel trajectories given by Theorem 3.1 continues to hold provided $N(a)=\mathrm{o}\left(a^{2}\right)$.

\section{B. Optimality for $\mathbb{R}^{d}$ where $d \geq 3$}

For fields in $\mathbb{R}^{d}$ with $d \geq 3$, we consider only trajectory sets of the form in (14). We also restrict ourselves to fields bandlimited to sets $\mho$ that are compact convex subsets of $\mathbb{R}^{d}$ and have a point of symmetry at the origin. As before, let $\mathcal{E}_{\mho}$ denote the class of trajectory sets $p \in \mathcal{C}_{\mho}$ that are composed of regular parallel trajectories expressed in the form (14). From Theorem 2.5 we know the necessary and sufficient conditions on the vectors $\left\{v^{1}, v^{2}, \ldots, v^{d}\right\}$ required for $p \in \mathcal{E}_{\mho}$. We now seek the solution to the problem

$$
\min _{p \in \mathcal{E}_{\mho}} \ell(p)
$$

where $\mho \subset \mathbb{R}^{d}$ is a compact convex set with a point of symmetry at the origin. In this section we outline a procedure for solving the above problem. In our approach we relate this problem to the problem of designing optimal sampling lattices for static sampling in $\mathbb{R}^{d-1}$.

Let $\left\{v^{1}, v^{2}, \ldots, v^{d}\right\}$ denote a basis for $\mathbb{R}^{d}$ and let $p$ be a trajectory set of the form (14). Let $U$ denote a $d \times d$ unitary matrix such that $U v^{d}=e^{d}$, the unit vector along the $d$-th principal axis. Define

$$
\hat{\mho}^{U}:=\left\{r \in \mathbb{R}^{d-1}: U^{-1}\left(\begin{array}{c}
r \\
0
\end{array}\right) \in \mho\right\} .
$$

Also let $\tilde{v}^{i}:=U v^{i}$ and $\breve{v}^{i}:=\left(\tilde{v}_{1}^{i}, \tilde{v}_{2}^{i}, \ldots \tilde{v}_{d-1}^{i}\right)^{T}$ denote the vector composed of the first $d-1$ components of $\tilde{v}^{i}$. Suppose $\left\{b^{1}, b^{2}, \ldots, b^{n}\right\}$ forms a basis for $\mathbb{R}^{n}$. A lattice $b$ of points in $\mathbb{R}^{n}$ of the form

$$
b=\left\{\sum_{i=1}^{n} m_{i} b^{i}: m \in \mathbb{Z}^{n}\right\}
$$

is called a sampling lattice for a set $\Phi \subset \mathbb{R}^{n}$ if every $n$ dimensional bandlimited field $g: \mathbb{R}^{n} \mapsto \mathbb{R}$ with Fourier transform supported on $\Phi$ can be recovered perfectly using only the values of the field $g$ measured at points in the lattice $b$. The sampling density of a sampling lattice of the form (21) is defined as the average number of points per unit volume in $\mathbb{R}^{n}$. Then we have the following result.

Theorem 3.2: Let $p$ be a trajectory set of the form in (14). Let $U, \hat{\mho}^{U}$ and $\breve{v}^{i}$ be as defined above. Then the trajectory set $p$ satisfies $p \in \mathcal{C}_{\mho}$ if and only if $b^{U}:=\left\{\sum_{i=1}^{d-1} m_{i} \breve{v}^{i}\right.$ : 
$\left.m \in \mathbb{Z}^{d-1}\right\}$ forms a sampling lattice for $\hat{\mho}^{U}$. Furthermore the path density $\ell(p)$ is equal to the sampling density of $b^{U}$. Proof: Consider the field $\tilde{f}$ defined by

$$
\tilde{f}(r)=f\left(U^{-1} r\right), r \in \mathbb{R}^{d} .
$$

Clearly the Fourier transform of $\tilde{f}$ is supported on the set

$$
\mho^{U}:=\{U s: s \in \mho\}
$$

Now the problem of sampling the field $f$ along trajectories $p_{m}(t)$ is equivalent to sampling the field $\tilde{f}$ along trajectories $\tilde{p}_{m}(t)$ defined by

$$
\tilde{p}_{m}(t)=\sum_{i=1}^{d-1} m_{i} \tilde{v}^{i}+t \tilde{v}^{d}, \quad t \in \mathbb{R}
$$

where $\tilde{v}^{i}=U v^{i}$. Hence if $\tilde{p}:=\left\{\tilde{p}_{m}: m \in \mathbb{Z}^{d-1}\right\}$ then it follows that

$$
p \in \mathcal{C}_{\mho} \quad \Leftrightarrow \quad \tilde{p} \in \mathcal{C}_{\mho U}
$$

and that the path density of $\tilde{p}$ is identical to that of $p$. We know from Theorem 2.5 that the necessary and sufficient condition for $\tilde{p} \in \mathcal{C}_{\mho^{U}}$ is given by

$$
\frac{1}{2} \sum_{i=1}^{d-1} m_{i} \tilde{u}^{i} \notin \mho^{U}, \text { for all } m \in\{-1,0,1\}^{d-1} \backslash\{0\}^{d-1}
$$

where $\tilde{u}^{i}$ are defined as vectors in $\mathbb{R}^{d}$ that satisfy $\left\langle\tilde{u}^{i}, \tilde{v}^{j}\right\rangle=$ $2 \pi \delta_{i j}$ for $1 \leq i \leq d-1$ and $1 \leq j \leq d$. Now since $\tilde{v}^{d}=e^{d}$, it follows that $\tilde{u}_{d}^{i}=0$ for $i \leq d-1$. Hence the condition (23) is equivalent to

$$
\frac{1}{2} \sum_{i=1}^{d-1} m_{i} \breve{u}^{i} \notin \hat{\mho}^{U}, \text { for all } m \in\{-1,0,1\}^{d-1} \backslash\{0\}^{d-1}
$$

where $\breve{u}^{i}:=\left(\tilde{u}_{1}^{i}, \tilde{u}_{2}^{i}, \ldots \tilde{u}_{d-1}^{i}\right)^{T}$. By construction it is clear that $\left\langle\breve{u}^{i}, \breve{v}^{j}\right\rangle=2 \pi \delta_{i j}$ for $1 \leq i, j \leq d-1$. Thus it follows from [1] that condition (24) is exactly the necessary and sufficient condition to ensure that $b^{U}$ forms a sampling lattice for $\hat{\mho}^{U}$.

We now consider the path density $\ell(\tilde{p})$. Since $\tilde{v}^{d}=e^{d}$ it is clear that the collection of points

$$
\left\{\sum_{i=1}^{d-1} m_{i} \tilde{v}^{i}+t \tilde{v}^{d}: m \in \mathbb{Z}^{d}, t \in \mathbb{R}\right\}
$$

remains unaltered if we replace $\tilde{v}^{i}$ by $\hat{v}^{i}:=\left(\begin{array}{c}\breve{v}^{i} \\ 0\end{array}\right)$ for $1 \leq i \leq d-1$. Thus it follows via Lemma 2.6 that the path density $\ell(\tilde{p})$ satisfies

$$
\ell(\tilde{p})=|\operatorname{det}(H)|^{-1}
$$

where $H$ is a $d-1 \times d-1$ matrix with entries $H_{i, j}=$ $\left\langle\hat{v}^{i}, \hat{v}^{j}\right\rangle=\left\langle\breve{v}^{i}, \breve{v}^{j}\right\rangle$. Since the sampling density of $b^{U}$ is equal to $|\operatorname{det}(H)|^{-1}$ (see [1]) and $\ell(p)=\ell(\tilde{p})$ the result follows.

As an immediate consequence of the above result we have the following corollary on the optimality of sampling trajectory sets from $\mathcal{E}_{\mho}$.
Corollary 3.2.1: Let $\mho \subset \mathbb{R}^{d}$ denote a compact convex set with a point of symmetry at the origin. Among all possible choices of $d \times d$ unitary matrices let $\widehat{U}$ be the one such that the set $\hat{\mho} \widehat{U}$ admits a sampling lattice with minimal sampling density in $\mathbb{R}^{d-1}$. Also suppose that the vectors $\left\{w^{1}, w^{2}, \ldots, w^{d-1}\right\} \subset \mathbb{R}^{d-1}$ generate an optimal sampling lattice for fields bandlimited to $\hat{\mho} \widehat{U}$. Let $p$ be a trajectory set as defined in (14) where the vectors $v^{i}$ are given by

$$
v^{i}=\widehat{U}^{-1} \check{w}^{i}, 1 \leq i \leq d-1 \text { and } v^{d}=\widehat{U}^{-1} e^{d}
$$

where $\check{w}^{i}=\left(w_{1}^{i}, w_{2}^{i}, \ldots, w_{d-1}^{i}, 0\right)^{T} \in \mathbb{R}^{d}$. Then $p \in \mathcal{E}_{\mho}$ and solves the optimization problem (19).

The problem of identifying sampling lattices with minimal density is well studied in the literature (see, e.g., [1], [14], [15]). Such results can be used in conjunction with the above corollary to design optimal sampling trajectories. In the following corollary we present a simple example of a field bandlimited to a spherical region for which we can explicitly identify the solution for the optimal sampling trajectory set.

Corollary 3.2.2: Suppose $\mho$ is a spherical ball in $\mathbb{R}^{d}$ with radius $\alpha$. Let $\left\{w^{1}, w^{2}, \ldots, w^{d-1}\right\} \subset \mathbb{R}^{d-1}$ generate a sampling lattice with minimal sampling density for fields bandlimited to a ball in $\mathbb{R}^{d-1}$ with radius $\alpha$. Let

$$
v^{i}:=\left(\begin{array}{c}
w^{i} \\
0
\end{array}\right), 1 \leq i \leq d-1 \quad v^{d}=e^{d} .
$$

Then the trajectory set $p$ defined in (14) with the above choices for vectors $v^{i}$ achieves the minimum in (19). For $d=3$ the vectors $v^{i}$ can be chosen as

$v^{1}=\frac{1}{\alpha}\left(\frac{\sqrt{3}}{2},-\frac{1}{2}, 0\right)^{T}, v^{2}=\frac{1}{\alpha}(1,0,0)^{T}, v^{3}=(0,0,1)^{T}$.

Proof: Since $\mho$ is a spherical ball in $\mathbb{R}^{d}$, it follows that $\hat{\mho}^{U}$ defined in (20) is a ball of radius $\alpha$ in $\mathbb{R}^{d-1}$ for all choices of the unitary matrix $U$. Hence without loss of optimality we choose $\widehat{U}$ in Corollary 3.2 .1 to be the identity matrix. Thus the first statement is immediate from Corollary 3.2.1. The second part follows from the fact that a sampling grid based on a $120^{\circ}$ rhombic lattice is optimal [1] for twodimensional isotropic fields.

We note that exact choices of vectors $v^{i}$ for fields bandlimited to spherical balls in $\mathbb{R}^{d}$ for all $d<8$ can be obtained from the above result using the results on optimal lattices for isotropic fields presented in [1, Table C.I]. As mentioned in [1], these results are based on results on closest packing of spheres in $\mathbb{R}^{d}$.

\section{RECONSTRUCTION SCHEMES}

We now consider schemes for reconstructing bandlimited fields using measurements of the field taken by sensors moving along the various trajectories we have considered so far. As we discussed earlier we know from classical sampling theory that if the trajectories satisfy condition (K1) then the readings over a discrete set of points are sufficient to be able to reconstruct values of bandlimited fields at all points on the sampling trajectories. We know from Lemma 2.2 that regular parallel trajectories of the form (4) for $\mathbb{R}^{2}$, or (8) 
with $N=1$ for $\mathbb{R}^{2}$, or (14) for $\mathbb{R}^{d}$, satisfy condition (K1). Hence the field values at all points on these trajectories can be reconstructed from samples taken at uniform spatial intervals. The maximum spacing allowed between adjacent samples on these trajectories can be calculated from Lemma 2.2. If we assume that the sensors on each of the parallel trajectories take samples at identical uniform intervals and that the sample locations on the various trajectories are aligned with each other, then the collection of all samples obtained on all these trajectories effectively corresponds to a collection of samples of the field taken over a periodic lattice of points. Hence, for such trajectory sets, any reconstruction algorithms used for reconstructing bandlimited fields on lattices is sufficient (see, e.g., [1], [2]). For unions of regular sets of parallel trajectories like in (8) the resulting set of points lie on a union of sampling lattices. In this case reconstruction schemes for sampling on unions of lattices are applicable (see, e.g., [9]).

Reconstruction schemes for the circular trajectories considered in Section II-B.3 are provided in [5] and [6]. However, since these trajectory sets do not satisfy condition (K1), the reconstruction schemes require the exact field values at all points on the circular trajectories, and unlike in the case of linear trajectories, the information necessary for reconstruction cannot be obtained by merely sampling along the sensor trajectories.

\section{CONCLUSION AND FUTURE WORK}

In this paper we have introduced the problem of sampling bandlimited fields in $\mathbb{R}^{d}$ on trajectories. We have presented various examples of sampling trajectories that admit perfect reconstruction of bandlimited fields, and some optimality properties of select trajectories in terms of the path density metric that we introduced.

This paper opens numerous avenues for future work in terms of extensions and generalizations. For instance, it would be of interest to ascertain the validity of the conjectures we mentioned in the end of Section III-A on the optimality of the regular set of parallel trajectories among a wider class of trajectory sets. Most results in this paper assume that the field is bandlimited to a convex subset of $\mathbb{R}^{d}$. It is of interest to study extensions to more general sets so as to address problems like that of sampling bandpass fields. It would also be of interest to study the design of sampling trajectories for non-bandlimited fields, e.g., spatial fields that form shift-invariant spaces or parametric fields like diffusion fields. In such contexts, the problem of optimizing the tradeoff between the total distance traveled by the sensors and the reconstruction accuracy is also a relevant problem. Another extension that we are currently studying is the design of sampling trajectories for time-varying bandlimited fields. We also have some ongoing work [8] on extensions of some of our results on sampling trajectories to higher dimensional sampling manifolds.

\section{APPENDIX}

\section{A. Proof of Lemma 2.1}

Let $p \in \mathcal{C}_{\mho}$. Then for every $i \in \mathbb{Z}$ and every $f \in \mathcal{B}_{\mho}$ we know that $f\left(p_{i}().\right)$ is bandlimited. In particular this holds true for fields of the form $f(r)=g\left(r_{i}\right)$ where $r_{i}$ is the $i$ the component of $r$, and $g$ is a function of one real variable which is bandlimited to $[-\Omega, \Omega]$ for some $\Omega>0$. Therefore if $p_{i}(t)=\left(p_{i, 1}(t), \ldots, p_{i, d}(t)\right)$ then for all such $g$ the function $g\left(p_{i, 1}().\right)$ is bandlimited. Under such a condition, it is known that $p_{i, 1}(t)$ must be an affine function of $t$ (see, e.g., [16] and [17]). By identical arguments it follows that $p_{i, j}(t)$ must also be an affine function of $t$ for every $i, j$.

\section{B. Proof of Lemma 2.2}

Let $F$ denote the Fourier transform of the field $f$. Then we have $f(p(t))=\frac{1}{(2 \pi)^{d}} \int_{\mho} F(\omega) \exp \left(\mathrm{i}\left\langle\omega, r^{0}+r^{1} t\right\rangle\right) d \omega$. Hence the Fourier transform $F_{p}$ of $f(p()$.$) is given by$

$$
\begin{aligned}
& F_{p}(s) \\
& =\frac{1}{(2 \pi)^{d}} \int_{\mathbb{R}} \int_{\mho} F(\omega) \exp \left(\mathrm{i}\left(\left\langle\omega, r^{0}+r^{1} t\right\rangle-s t\right)\right) d \omega d t \\
& =\frac{1}{(2 \pi)^{d-1}} \int_{\mho} F(\omega) \exp \left(\mathrm{i}\left\langle\omega, r^{0}\right\rangle\right) \delta\left(\left\langle\omega, r^{1}\right\rangle-s\right) d \omega
\end{aligned}
$$

and thus $F_{p}(s)=0$ for $s \notin[\underline{\Omega}, \bar{\Omega}]$.

\section{Proof of Lemma 2.3}

Consider a disc of radius $a$ centered at the origin. Let $\ell_{i}$ denote the length of the intersection of the disc with the trajectory $p_{i}$ defined in (4). Let $N \approx \frac{a}{\alpha}$ denote the highest index of the trajectories with non-zero intersection with the disc. Now we can approximate the area of the disc above and below by rectangular regions with sides $\ell_{i} \times \alpha$ to obtain the inequality:

$$
2 \sum_{i=1}^{N} \ell_{i} \alpha \leq \pi a^{2} \leq 2 \sum_{i=0}^{N} \ell_{i} \alpha .
$$

Now $\mathcal{D}^{p}(a)=\ell_{0}+2 \sum_{i=1}^{N} \ell_{i}$. Hence we have

$$
\mathcal{D}^{p}(a)-\ell_{0} \leq \frac{\pi a^{2}}{\alpha} \leq \mathcal{D}^{p}(a)+\ell_{0}
$$

and (5) follows by taking limits.

\section{Outline of proof of Lemma 2.6}

Lemma 2.6 can be proved using the same approach as in the proof of Lemma 2.3. Just as we approximated a circle with rectangles in the proof of Lemma 2.3 we will now approximate a $d$-spherical ball with parallelotopes. Let $\mathcal{S}_{a}^{d}$ denote a $d$-dimensional spherical ball of radius a centered at the origin in $\mathbb{R}^{d}$. For $r \in \mathbb{R}^{d}$ let $\beta_{i}(r)$ denote the coefficients in the basis expansion $r=\sum_{i=1}^{d} \beta_{i}(r) v^{i}$. Now for $m \in \mathbb{Z}^{d-1}$ define

$$
S_{m}:=\left\{x \in \mathcal{S}_{a}^{d}: \beta_{i}(x) \in\left[m_{i}, m_{i}+1\right), 1 \leq i \leq d-1\right\} .
$$


Let

$$
M_{a}:=\left\{m \in \mathbb{Z}^{d-1}: p_{m}(t) \in \mathcal{S}_{a}^{d} \text { for some } t \in \mathbb{R}\right\}
$$

with $p_{m}(t)=\sum_{i=1}^{d-1} m_{i} v^{i}+t v^{d}$ as defined in (13). For each $m \in M_{a}$ let $\ell_{m}(a)$ denote the length of the line segment representing the intersection of trajectory $p_{m}($.$) with \mathcal{S}_{a}^{d}$. We then have

$$
\operatorname{Vol}\left(\mathcal{S}_{a}^{d}\right)=\sum_{m \in M_{a}} \operatorname{Vol}\left(S_{m}\right)+\mathrm{o}\left(a^{d-1}\right) .
$$

Now we can approximate $S_{m}$ with the parallelotope generated by the vectors $\left\{v^{1}, v^{2}, \ldots, v^{d-1}, \frac{\ell_{m}(a)}{\left\|v^{d}\right\|} v^{d}\right\}$ so that $\operatorname{Vol}\left(S_{m}\right) \approx \frac{|\operatorname{det}(G)|}{\left\|v^{d}\right\|} \ell_{m}(a)$. In fact it can be shown that

$$
\sum_{m \in M_{a}} \operatorname{Vol}\left(S_{m}\right)=\sum_{m \in M_{a}} \frac{|\operatorname{det}(G)|}{\left\|v^{d}\right\|} \ell_{m}(a)+\mathrm{o}\left(a^{d-1}\right) .
$$

Now $\sum_{m \in M_{a}} \ell_{m}(a)=\mathcal{D}^{p}(a)$ and hence the result follows.

\section{E. Proof of Theorem 3.1}

From Theorem 2.4 it easily follows that $p^{\epsilon} \in \mathcal{D}_{\mho}$. Suppose $p^{\epsilon}$ is expressed in the form $\hat{p}^{1}$ as defined in (8) with $\alpha=1$. Then the vector $u^{1}$ appearing in Theorem 2.4 corresponding to $\hat{p}^{1}=p^{\epsilon}$ is given by

$$
u^{1}=\left(1-\frac{\epsilon \mathcal{W}(\mho)}{2 \pi}\right)^{-1} \hat{u}
$$

and hence by the definition of $\hat{u}$ and by (11) we have $p^{\epsilon} \in$ $\mathcal{D}_{\mho}$ for all $\epsilon>0$. Also from (9) we have

$$
\lim _{\epsilon \rightarrow 0} \ell\left(p^{\epsilon}\right)=\frac{2 \pi}{\mathcal{W}(\mho)} \text {. }
$$

In order to complete the proof we will establish that for all $p \in \mathcal{D}_{\mho}$, we have $\ell(p) \geq \frac{2 \pi}{\mathcal{W}(\mho)}$. Any trajectory set in $\mathcal{D}_{\mho}$ can be expressed in the form $\hat{p}^{1}$ as defined in (8) with $\alpha=1$. Using the notations and assumptions of Theorem 2.4 it follows that if $\hat{p}^{1} \in \mathcal{D}_{\mho}$ we need

$$
\mathcal{Q}^{1} \nsubseteq \stackrel{i}{\mho}(r) \text { for all } r \in \mathbb{R}^{2}
$$

where $i(r)$ denotes the interior of set $\mho(r)$. Together with the relation (18) satisfied by the width of a convex set, it follows that the length of some diagonal in the parallelotope $\mathcal{Q}^{1}$ must be greater than $\mathcal{W}(\mho)$. This means that

$\left\|\sum_{i=1}^{N}(-1)^{n_{i}} u^{i}\right\| \geq \mathcal{W}(\mho)$ for some $n_{i} \in\{0,1\}, 1 \leq i \leq N$.

This means that the path density defined in (9) satisfies

$$
\begin{aligned}
\ell\left(\hat{p}^{1}\right) & =\frac{1}{2 \pi} \sum_{i=1}^{N}\left\|u^{i}\right\| \geq \frac{1}{2 \pi}\left\|\sum_{i=1}^{N}(-1)^{n_{i}} u^{i}\right\| \\
& \geq \frac{\mathcal{W}(\mho)}{2 \pi} .
\end{aligned}
$$

\section{REFERENCES}

[1] D. P. Petersen and D. Middleton, "Sampling and Reconstruction of Wave-Number-Limited Functions in N-Dimensional Euclidean Spaces," Inform. Contr., vol. 5, pp. 279-323, 1962.

[2] K. Grochenig, "Reconstruction algorithms in irregular sampling," Mathematics of Computation, vol. 59, no. 199, pp. pp. 181-194, 1992. [Online]. Available: http://www.jstor.org/stable/2152989

[3] A. Cigada, M. Lurati, F. Ripamonti, and M. Vanali, "Moving microphone arrays to reduce spatial aliasing in the beamforming technique: theoretical background and numerical investigation," J. Acoust. Soc. Am, vol. 124, no. 6, pp. 3648-3658, 2008.

[4] J. Unnikrishnan and M. Vetterli, "Sampling and reconstructing spatial fields using mobile sensors," 2011, submitted to IEEE Int. Conf Acoust., Speech, and Signal Proc.,2012.

[5] A. Tewfik, B. Levy, and A. Willsky, "Sampling theorems for twodimensional isotropic random fields," IEEE Trans. Inf. Theory, vol. 34, no. 5, pp. 1092 -1096, Sep. 1988.

[6] N. E. Myridis and C. Chamzas, "Sampling on concentric circles." IEEE Trans. Med. Imaging, pp. 294-299, 1998.

[7] M. T. Vlaardingerbroek and J. A. den Boer, Magnetic Resonance Imaging: Theory and Practice. New York: Springer, 2003.

[8] J. Unnikrishnan and M. Vetterli, "Sampling manifolds for bandlimited fields," 2011, under preparation.

[9] H. Behmard and A. Faridani, "Sampling of bandlimited functions on unions of shifted lattices," J. Fourier Anal. Appl, vol. 8, pp. 43-58, 2002.

[10] A. Papoulis, "Generalized sampling expansion," Circuits and Systems, IEEE Transactions on, vol. 24, no. 11, pp. 652 - 654, Nov 1977.

[11] K. Gröchenig and H. Razafinjatovo, “On Landau's necessary density conditions for sampling and interpolation of bandlimited functions," Journal of the London Mathematical Society, vol. 54, no. 3, pp. 557-565, 1996. [Online]. Available: http://jlms.oxfordjournals.org/content/54/3/557.abstract

[12] F. A. Valentine, Convex Sets. New York: McGraw-Hill, 1964.

[13] H. Landau, "Necessary density conditions for sampling and interpolation of certain entire functions," Acta Mathematica, vol. 117, no. 1, pp. 37-52, Jul. 1967. [Online]. Available: http://dx.doi.org/10.1007/BF02395039

[14] Y. Lu, M. Do, and R. Laugesen, "A computable Fourier condition generating alias-free sampling lattices," IEEE Trans. Signal Process., vol. 57, no. 5, pp. $1768-1782$, may 2009.

[15] R. Mersereau, "The processing of hexagonally sampled twodimensional signals," Proceedings of the IEEE, vol. 67, no. 6, pp. 930 - 949, June 1979

[16] M. Do, D. Marchand-Maillet, and M. Vetterli, "On the bandwidth of the plenoptic function," Image Processing, IEEE Transactions on, vol. PP, no. 99, p. 1, 2011.

[17] S. Azizi, D. Cochran, and J. N. McDonald, "On the preservation of bandlimitedness under non-affine time warping," in Proc. Int. Workshop on Sampling Theory and Applications, August 1999. 